\title{
First Presentation of Systemic Lupus Erythematosus in a 24-Year-Old Male following mRNA COVID-19 Vaccine
}

\author{
Yael Raviv $\mathbb{D}^{1},{ }^{1}$ Batya Betesh-Abay, ${ }^{2}$ Yuliya Valdman-Grinshpoun, ${ }^{3}$ Liora Boehm-Cohen, ${ }^{1}$ \\ Michael Kassirer, ${ }^{1}$ and Iftach Sagy ${ }^{4}$ \\ ${ }^{1}$ Pulmonary Institute, Soroka University Medical Center, Beer-Sheva, Israel \\ ${ }^{2}$ Recanati School for Community Health Professions, Department of Nursing, Faculty of Health Sciences, \\ Ben-Gurion University of the Negev, Israel and Soroka University Medical Center, Beer-Sheva, Israel \\ ${ }^{3}$ Department of Dermatology, Soroka University Medical Center, Beer Sheva, Israel \\ ${ }^{4}$ Rheumatology Unit and Clinical Research Center, Soroka University Medical Center, Beer-Sheva, Israel \\ Correspondence should be addressed to Yael Raviv; yaelr2@clalit.org.il
}

Received 14 November 2021; Accepted 8 January 2022; Published 1 February 2022

Academic Editor: Tsai Ching Hsu

Copyright (c) 2022 Yael Raviv et al. This is an open access article distributed under the Creative Commons Attribution License, which permits unrestricted use, distribution, and reproduction in any medium, provided the original work is properly cited.

\begin{abstract}
The SARS-CoV-2 viral pandemic has had an immeasurable global impact, resulting in over 5 million deaths worldwide. Numerous vaccines were developed in an attempt to quell viral dissemination and reduce symptom severity among those infected. Systemic lupus erythematosus (SLE) is an autoimmune disease characterized by the production of antinuclear autoantibodies (ANAs) with heterogenic clinical manifestations, secondary to immune complex deposition in a multitude of organ systems. There are scarcely reported cases of SLE development following COVID-19 mRNA vaccination. We present a case of a 24-year-old male without preexisting conditions or family history of autoimmune disorders, presenting with SLE following the first dose of the SARS-CoV-2 Pfizer-BioNTech mRNA vaccine.
\end{abstract}

\section{Introduction}

Systemic lupus erythematosus (SLE) is an autoimmune disease characterized by the presence of antinuclear antibodies (ANA) and formation of immune complexes, which can directly and indirectly cause inflammatory damage to multiple organs [1]. While the prevalence of SLE is the highest among reproductive age, nonwhite women, SLE can be manifested virtually at any age, gender, or ethnic group [2]. Patients with SLE demonstrate lower cytotoxic $T$ and CD4 T lymphocyte levels, both integral modulators of the humeral immune response $[3,4]$.

The SARS-CoV-2 viral pandemic has had an immeasurable global impact, responsible for over 5 million deaths worldwide [5]. Numerous vaccines were developed in attempt to quell viral dissemination and reduce symptom severity among those infected. The Pfizer-BioNTech BNT162b2 vaccine displayed exceptional safety and efficacy in clinical trials and in postrelease monitoring [6], and Israel was among the first countries to achieve mass immunization [7, 8]. Although Barda et al. [9] evaluated the broad range of potential adverse events documented following the BNT162b2 mRNA COVID-19 vaccine in a nationwide setting, autoimmune diseases were excluded from the analysis owing to the shortened reporting time period (42 days) deemed to be too brief for the development of autoimmune diseases [9]. Long-term (i.e., greater than one year) and rare adverse effects of the vaccine have not been fully elucidated. Studies have reviewed autoimmune dysregulations in patients with COVID-19 [10-12], and there is a paucity of studies exploring patients with prediagnosed SLE and their tolerability and susceptibility contextualized around receiving the COVID-19 mRNA vaccine $[13,14]$. However, minimal reportings have described the development of SLE first erupting following the COVID-19 mRNA vaccination. 


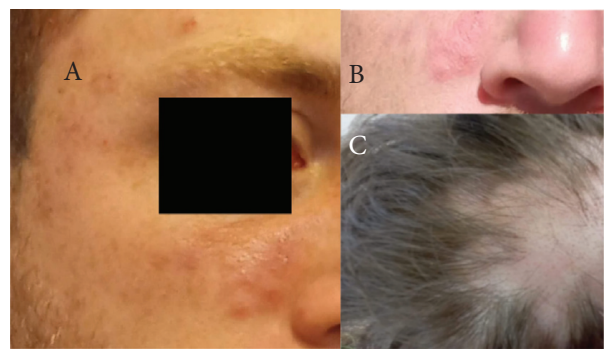

Figure 1: 24-year-old male patient 62 days following vaccine administration. (a, b) Psoriasiform-papulosquamous plaques over the face. (c) Nonscarring hair loss over the head.

TABLe 1: Chronology of clinical manifestations, laboratory results, and treatment.

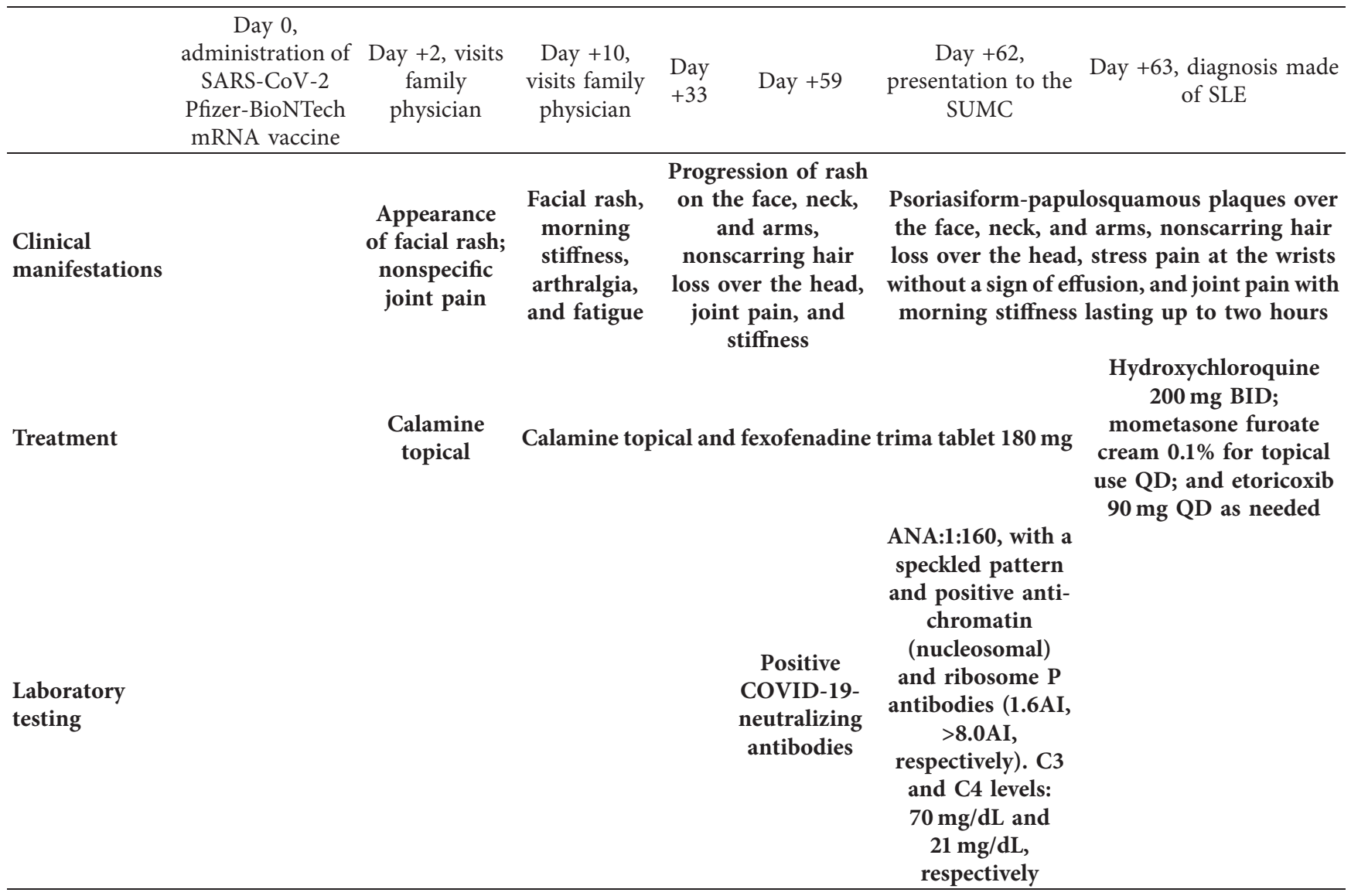

ANA: antinuclear antibody, SLE: systemic lupus erythematosus, and SUMC: Soroka University Medical Center.

\section{Case Report}

A 24-year-old Israeli male of Ashkenazi descent, with no premorbidities or family history of autoimmune conditions, visited his family physician two days following administration of the first dose of the SARS-CoV-2 Pfizer-BioNTech mRNA vaccination complaining of a facial rash. He was prescribed calamine lotion for a suspected allergic reaction. The patient's environmental exposures included outdoor agricultural work and daily smoking. He did not drink alcohol or use illicit drugs. The patient subsequently returned to his physician ten days later due to progression and worsening of the rash, and fexofenadine trima tablet $180 \mathrm{mg}$ was added.

The patient presented to the Soroka University Medical Center (SUMC) 8 weeks later due to continued symptoms. At that time, the patient described multiple joint pains with morning stiffness lasting up to two hours. Dermatologist evaluation was significant for psoriasiform-papulosquamous plaques over the face, neck, and arms, nonscarring hair loss over the head (Figure 1), and bilateral wrist pain without evidence of swelling. There was normal lung sound on auscultation, without oral/ nasal ulcers, xerostomia, or keratoconjunctivitis sicca. 
Initial laboratory results demonstrated normal range complete blood count, serum chemistries, C-reactive protein, and erythrocyte sedimentation rate. Serology testing was positive for antinuclear antibody (ANA) (1: 160) with a speckled pattern and positive antichromatin (nucleosomal) and ribosome $\mathrm{P}$ antibodies (1.6 AI and $>8.0$ AI, respectively). Anti-double-stranded deoxyribonucleic acid, anti-Smith, SS-A, and SS-B antibodies were all negative. The C3 and C4 levels were $70 \mathrm{mg} / \mathrm{dL}$ and $21 \mathrm{mg} /$ $\mathrm{dL}$, respectively. Anti-cardiolipin antibodies and beta-2glycoprotein antibodies (IgM and IgG for both), in addition to lupus anticoagulant, were negative. The direct Coombs test was also negative. Urinalysis was without casts, hematuria, or proteinuria. Table 1 presents the chronology of symptomatic manifestations, treatment, and laboratory findings accordingly.

A diagnosis of SLE was established based on the 2019 American College of Rheumatology/European League Against Rheumatism (ACR/EULAR) classification criteria [15]. Hydroxychloroquine $200 \mathrm{mg}$ BID, mometasone furoate cream $0.1 \%$, and etoricoxib $90 \mathrm{mg}$ QD as needed were initiated. The patient was instructed to avoid direct exposure to sun and to use broad-spectrum sunscreens.

The patient was recommended not to receive the second dose of the SARS-CoV-2 Pfizer-BioNTech mRNA vaccine. He was found to have COVID-19-neutralizing antibodies in the follow-up serology testing.

The patient responded well to treatment and demonstrated improvement in subsequent visits, including the disappearance of rash.

\section{Discussion}

In the case study described above, a patient exhibited an initial presentation of SLE following the mRNA COVID-19 vaccine. The exact etiology of SLE is likely multifactorial but still only incompletely elucidated. No correlation between mRNA COVID-19 vaccine administration and the imminent development of SLE has been reported. The clinical manifestations of SLE, such as nephritis, pleuritis, cardiomyopathy, and dermatitis, are mediated by antibody formation and the creation of immune complexes, ultimately causing small-vessel vasculitis. Studies have demonstrated the critical role of B-cell hyperactivity and excessive autoantibody production in SLE pathophysiology [16]. Additionally, the activation of CD4+ immune pathways has also been demonstrated in the pathophysiology of SLE. Zhao et al. demonstrated the role of microRNA in regulating DNA methylation in CD4+ T cells and its contribution to T-cell autoreactivity in SLE [17]. Additionally, the Ro/SS-A antigen is a $60 \mathrm{kDa}$ protein attached to small RNAs to produce a $100 \mathrm{kDa}$ complex with a nuclear localization in the skin, which can lead to adult onset of subacute cutaneous lupus erythematosus (SCLE) and neonatal lupus [18]. Hence, there is a plausible CD4+ involved in the pathogenesis of SLE that could be the biological mechanisms activated by the mRNA COVID19 vaccine.

The notion of vaccine-induced autoimmune response has been suggested in the context of immune thrombocytopenia, autoimmune myocarditis, and autoimmune hepatitis [19-21]. While the temporal relationship may be coincidental, it is feasible that vaccine administration stimulates unregulated immunogenicity among subjects with a specific predisposition.

\section{Conclusions}

While the SARS-CoV-2 Pfizer-BioNTech mRNA vaccination has thus far demonstrated outstanding efficacy and safety, rare and long-term side effects are still not known. Further research is needed to determine if the PfizerBioNTech mRNA vaccine can cause untoward immune modulatory effects, including the development of autoimmune disorders such as SLE.

\section{Data Availability}

Data were ethically extracted from the patient's file.

\section{Ethical Approval}

This study received exemption of ethical approval from the Helsinki Review Committee of SUMC, Israel.

\section{Consent}

Written informed consent was obtained from the patient described for the publication of this case report as well as the provided images.

\section{Disclosure}

Y.R and B.B-A should be considered equal first authors.

\section{Conflicts of Interest}

The authors declare no conflicts of interest.

\section{Authors' Contributions}

(1) Y.R and B.B-A contributed equally to this study. (2) Y.R collected the clinical data, treated the patient, and conducted overall supervision and review of the manuscript. B.B-A assisted in data collection, prepared the figure and table, and formatted and wrote original draft of the manuscript. Y. V-G, L. B-C, and M.K helped supervise the project. I.S treated the patient and contributed to drafting and writing of introduction, case report, and discussion. All authors discussed the results, provided critical feedback, and contributed to the final draft and have read and agreed to the published version of the manuscript.

\section{References}

[1] T. Dörner and R. Furie, "Novel paradigms in systemic lupus erythematosus," Lancet, vol. 393, pp. 2344-2358, 2019.

[2] G. J. Pons-Estel, G. S. Alarcón Graciela, L. Scofield, L. Reinlib, and G. S. Cooper, "Understanding the epidemiology and progression of systemic lupus erythematosus," Seminars in Arthritis and Rheumatism, vol. 39, no. 4, pp. 257-268, 2010.

[3] J. Yang, X. Yang, H. Zou, Y. Chu, and M. Li, "Recovery of the immune balance between Th17 and regulatory $\mathrm{T}$ cells as a 
treatment for systemic lupus erythematosus," Rheumatology, vol. 50, no. 8, pp. 1366-1372, 2011.

[4] M. Miyara, Z. Amoura, C. Parizot et al., "Global natural regulatory $\mathrm{T}$ cell depletion in active systemic lupus erythematosus," The Journal of Immunology, vol. 175, no. 12, pp. 8392-8400, 2005.

[5] WHO Coronavirus (COVID-19) Dashboard, https://covid19. who.int/.

[6] F. P. Polack, S. J. Thomas, N. Kitchin et al., "Safety and efficacy of the BNT162b2 mRNA covid-19 vaccine," New England Journal of Medicine, vol. 383, no. 27, pp. 2603-2615, 2020.

[7] B. Rosen, R. Waitzberg, and A. Israeli, "Israel's rapid rollout of vaccinations for COVID-19," Israel Journal of Health Policy Research, vol. 10, no. 1, 6 pages, 2021.

[8] E. Mathieu, H. Ritchie, E. Ortiz-Ospina et al., "A global database of COVID-19 vaccinations," Nature Human Behaviour, vol. 5, no. 7, pp. 947-953, 2021.

[9] N. Barda, N. Dagan, and Y. Ben-Shlomo, "Safety of the BNT162b2 mRNA COVID-19 vaccine in a nationwide setting," New England Journal of Medicine, vol. 385, pp. 10781090, 2021.

[10] A. Vojdani and D. Kharrazian, "Potential antigenic crossreactivity between SARS-CoV-2 and human tissue with a possible link to an increase in autoimmune diseases," Clinical Immunology, vol. 217, Article ID 108480, 2020.

[11] M. Ehrenfeld, A. Tincani, L. Andreoli et al., "Covid-19 and autoimmunity," Autoimmunity Reviews, vol. 19, no. 8, Article ID 102597, 2020.

[12] Y. Liu, A. H. Sawalha, and Q. Lu, "COVID-19 and autoimmune diseases," Current Opinion in Rheumatology, vol. 33, no. 2, pp. 155-162, 2021.

[13] W. Tang, A. D. Askanase, L. Khalili, and J. T. Merrill, "SARSCoV-2 vaccines in patients with SLE," Lupus Science \& Medicine, vol. 8, no. 1, Article ID e000479, 2021.

[14] A. Mason, H. Anver, and M. Lwin, "Lupus, vaccinations and COVID-19: what we know now," Lupus, vol. 30, no. 10, pp. 1541-1552, 2021.

[15] M. Aringer, K. Costenbader, D. Daikh et al., "2019 European League against Rheumatism/American College of Rheumatology classification criteria for systemic lupus erythematosus," Annals of the Rheumatic Diseases, vol. 78, pp. 1151-1159, 2019.

[16] A. Sang, Y.-Y. Zheng, and L. Morel, "Contributions of B cells to lupus pathogenesis," Molecular Immunology, vol. 62, no. 2, pp. 329-338, 2014.

[17] S. Zhao, Y. Wang, Y. Liang et al., "MicroRNA-126 regulates DNA methylation in CD4+ T cells and contributes to systemic lupus erythematosus by targeting DNA methyltransferase 1," Arthritis \& Rheumatism, vol. 63, no. 5, pp. 1376-1386, 2011.

[18] A. N. Crowson and C. Magro, "The cutaneous pathology of lupus erythematosus: a review," Journal of Cutaneous $\mathrm{Pa}$ thology, vol. 28, no. 1, pp. 1-23, 2001.

[19] E. Lee, D. B. Cines, and T. Gernsheimer, "Thrombocytopenia following pfizer and moderna SARS-CoV-2 vaccination," American Journal of Hematology, vol. 96, no. 5, pp. 534-537, 2021.

[20] B. Chamling, V. Vehof, and S. Drakos, "Occurrence of acute infarct-like myocarditis following COVID-19 vaccination: just an accidental co-incidence or rather vaccination-associated autoimmune myocarditis?" Clinical Research in Cardiology, vol. 110, no. 11, pp. 1850-1854, 2021.

[21] F. Bril, S. Al Diffalha, and M. Dean, "Autoimmune hepatitis developing after coronavirus disease 2019 (COVID-19) vaccine: causality or casualty?" Journal of Hepatology, vol. 75, no. 1, pp. 222-224, 2021. 